\title{
HIPPARCOS: ITS LINK TO AN EXTRAGALACTIC REFERENCE FRAME
}

\author{
A.N. ARGUE \\ Institute of Astronomy \\ University of Cambridge \\ Madingley Road \\ Cambridge CBS OHA, UK.
}

ABSTRACT. This paper reviews the links currently envisaged for a mission of duration $3 \frac{1}{2}$
years, with estimates of the precision of each link.

\section{Introduction}

The Catalogue of star coordinates to be produced by HIPPARCOS will be highly rigid and homogeneous, but will have arbitrary zero-points because no observations are being made by which the mean equator and the dynamical equinox could be located directly. In the first instance the Catalogue, comprising about 120,000 stars, will be aligned to FK5 by the two Data Analysis Consortia as an off-line task, working independently. Then, if data from the so-called 'Super-high Priority Link' (Section 2) are ready in time, the alignment will be further refined; if not, the FK5-aligned Catalogue will be published as it stands, and further refinement by the methods of Sections 2, 3 and 4 carried out subsequently. The ultimate purpose is to establish a conventional celestial barycentric reference frame, as close as possible to the existing FK5 equator and equinox referred to $\mathrm{J} 2000.0$, and accessible to astrometry at visual and radio frequencies, in compliance with the recommendation of the IAU WG on Reference Systems made at this Colloquium.

The VLBI Reference Frame due to be presented to the IAU General Assembly in 1994 will be made up from a number of tentative frames combined in the manner now carried out by IERS. At present, the right ascension scales of these frames are purely relative scales, and alignment to the FK5 right ascension scale is done through optical astrometry of some tens of quasars (Feissel, 1990. The practice of using one object alone, such as Algol or $3 \mathrm{C273B}$, has largely been discontinued). The FK5 frame is known to contain regional warps (Morrison et al., 1990), so that HIPPARCOS will give a better realization of the FK5 system in RA than the VLBI frames. In RA the link will thus be in the sense of linking VLBI to HIPPARCOS-FK5. Thereafter the RA zero-point will be a non-rotating vector locked into the extragalactic frame. The question of whether this frame does or does not possess a cosmic rotation, is not one that will be considered here.

The accuracies of the HIPPARCOS positions and proper motions for the $3 \frac{1}{2}$ year mission are estimated at $\pm 0^{\prime \prime} .002$ and $\pm 0^{\prime \prime} .002 \mathrm{yr}^{-1}$ respectively. It would be desirable if the rms error of the rotation matrix $R$ and its time derivative $R^{\prime}$ applied to the HIPPARCOS-FK5 
frame to link to VLBI, be not greater than one or two tenths of these quantities. The rms error in $R$ specifies the accuracy with which the optical and radio frames have been unified at the specified epoch, while that of $R^{\prime}$ gives limits to their relative rotation.

\section{The Super-high Priority Link}

Two methods have been selected: (i) Hubble Space Telescope and (ii) Radio Stars.

\subsection{HUBBLE SPACE TELESCOPE}

The intention is to measure the separation, and its rate of change with time, between pairs of quasars and HIPPARCOS stars, both situated within the same 'pickle' of the Fine Guidance Sensor. Unfortunately so many difficulties have arisen that the schedule of Hubble observations for this program has had to be suspended for the present (Jefferys et al., 1990).

\subsection{RADIO STARS}

Many radio stars are sufficiently bright to be well within the range of accurate measurement by HIPPARCOS (the limit is about $11^{m}$, but the accuracy improves with increasing brightness). Radio stars are not extragalactic, so that measurements must be repeated over suitable timescales to obtain their proper motions. In addition, their radio fluxes are highly variable and usually very faint (below a few $\mathrm{mJy}$ at $5 \mathrm{GHz}$ ), but many are amenable to phase-reference relative to VLBI reference frame quasars situated within a few degrees on the sky. Because of their extreme variablity, VLBI observations can accumulate only rather slowly. Some radio stars such as Algol display finite structure so that a series of measurements is needed in order to model the offset between the optical and radio emitting regions at the epoch of each HIPPARCOS observation (Lestrade et al., 1990). But the selection has been weighted in favour of RS CVn binaries which are more compact: many are resolved only at the $0^{\prime \prime} .002$ level.

The programs to be described are drawn from only a small fraction of those stars that have confirmed radio emission and are included in the HIPPARCOS Input Catalogue (there are 186 in the Super-high Priority Link alone) but any new programs commencing only now would probably not be completed in time for that link. An inventory of radio stars has been compiled by Walter (1990).

2.2.1. NRL/USNO/CSIRO. This program consists of 54 stars north of $\delta=-26^{\circ}$ with plans for a southern extension, giving altogether about 100 stars (De Vegt et al., 1990).

2.2.2. JPL. Eleven $\operatorname{stars}^{1}$ north of $\delta=0^{\circ}$ (Lestrade et al., 1990).

2.2.3. MERLIN (Multi-Element Radio Linked Interferometer Network, Jodrell Bank). Twelve stars $^{2}$ have been accepted for measurement (Anderson et al., 1989).

\footnotetext{
${ }^{1}$ LS161 303; Algol; UX Ari; HR1099; FK Com; HR5110; бCrB; Cyg X1; AR Lac; SZ Psc; II Peg.

${ }^{2}$ HD1061; UX Ari; HD26337; b Per; 54 Cam; BD+52 ${ }^{\circ} 1579 ; \kappa$ Dra; 55 Boo; HD341475; BD $+43^{\circ} 3571$;
} 
2.2.4. Accuracy of Link. If at the end of the mission 50 radio stars have been measured by HIPPARCOS and VLBI, all to the same accuracy of $0^{\prime \prime} .002$ in position and $0^{\prime \prime} 002 \mathrm{yr}^{-1}$ in proper motion, in both optical and radio, the rms errors in $R$ and $R^{\prime}$ will be:

$\sigma_{R}=0^{\prime \prime} .0005$ and $\sigma_{R^{\prime}}=0^{\prime \prime} .0007 \mathrm{yr}^{-1}$ (Froeschlé and Kovalevsky, 1982).

\section{Ground-based Optical Astrometry of Quasars}

The brightness of the optical counterparts of the VLBI quasars runs from about $15^{\mathrm{m}}$ to fainter, and are well below the range of HIPPARCOS ${ }^{3}$. The resolution needed to relate the radio and optical feature in a quasar requires an optical telescope of aperture at least $2 \mathrm{~m}$, which means in practice that the field size is limited to a diameter of order $1^{\circ}$, and this is not sufficient to include enough HIPPARCOS stars for a link (on average there are fewer than 3 stars per $\square^{\circ}$ ). So the step from HIPPARCOS to quasar must be bridged by one or two intermediate steps provided by wide field astrographs. These also reduce the difficulties encountered in the photography over such large brightness ranges. The precision of a single object is about 15 times rms worse than the HIPPARCOS and VLBI precision, so to obtain accuracies in $R$ and $R^{\prime}$ that are comparable to those anticipated for the Super-high Priority Link, it is necessary to increase both the time base and the number of quasars. For example, a 5-year program of 400 quasars would yield:

$$
\sigma_{R}=0^{\prime \prime} .0019 \text { and } \sigma_{R^{\prime}}=0^{\prime \prime} .0006 y r^{-1} \text {. }
$$

No programs have yet been offered involving ground-based interferometry or CCDs used in the manner of the Hubble pickle: undoubtedly such methods will play important roles in the future, but are unlikely to be ready in time for the present mission.

\subsection{LARGE TELESCOPE PROGRAMS}

3.1.1. NRL/HAMBURG/NASA/GSFC/CSIRO/USNO. This 5-year program was commenced in 1987, and is described by Russell et al. (1990). The aim is for 400 sources with optical counterparts suitable for astrometry, distributed over the whole sky.

3.1.2. CONFOR. A Russian program intended for completion by 1992. It is described by Kumkova et al. (1990). There are about 200 sources with suitable optical counterparts, mostly north of $\delta=-26^{\circ}$.

\section{2. $\quad$ FICTITIOUS PROPER MOTIONS OF QUASARS}

A program described by Brosche et al. (1990), based on plates some as old as 90 years, with

${ }^{8} 3$ C273B was measured on 4 th. July 1990 , but the signal-to-noise ratio was insufficient for the measurements to play any significant role in the link.
} 
about 10 quasars. A preliminary reduction for four quasars ${ }^{4}$ gives for the three components of the rotational link:

$$
\sigma\left(\omega_{1}\right)=0^{\prime \prime} .0010 y r^{-1} ; \sigma\left(\omega_{2}\right)=0^{\prime \prime} .0010 y r^{-1} ; \sigma\left(\omega_{3}\right)=0^{\prime \prime} .0014 y r^{-1}
$$

\section{Absolute Proper Motion Surveys}

Provision has been made in the HIPPARCOS Input Catalogue for three surveys of proper motion of stars measured relative to galaxies, as follows:

\subsection{LICK NPM}

This survey contains about 300,000 stars in the brightness range $B=9^{m}$ to $18^{m}$ north of $\delta=-23^{\circ}$ (Klemola, 1990). The rms error of a single proper motion is $0^{\prime \prime} .005 \mathrm{yr}^{-1}$. There are about 19,000 stars in common with the HIPPARCOS Input Catalogue, of which 14,100 are from AGK3 and the remainder, all south of $\delta=-2^{\circ}$, from SAO.

\subsection{YALE-SAN JUAN SPM}

This is similar to the Lick Survey of which it is a southern extension, but because the measuring phase had not been entered until after the HIPPARCOS Input Catalogue had reached an advanced stage, it was possible to make a more link-oriented selection of stars. Selection was based mainly on the SIMBAD Database, and there are about 12,000 stars in common with the HIPPARCOS Input Catalogue (Van Altena et al., 1990).

Combining both the NPM and the SPM Surveys into one single all-sky survey, the formal rms error for $R^{\prime}$, ignoring possible zonal errors, is $0^{\prime \prime} .00007 \mathrm{yr}^{-1}$.

\subsection{TAUTENBURG}

This program uses the Tautenburg Schmidt Telescope and the ASCORECORD, APM (Cambridge) and MAMA (Paris) measuring machines. The present status is 15 link fields with at least 250 HIPPARCOS stars, giving an anticipated $\sigma_{R^{\prime}}=0^{\prime \prime} .004 \mathrm{yr}^{-1}$. Preliminary details have been published by Dick et al. (1987).

\section{Appendix}

The members of the HIPPARCOS Input Catalogue Consortium WG on the Extragalactic Link are:

A.N. Argue (Cambridge, Chairman); P. Brosche (Bonn); C. De Vegt (Hamburg); R.L. Duncombe (Austin); P.D. Hemenway (Austin); A.R. Klemola (Lick); J. Kovalevsky (CERGA); J.-F. Lestrade (Paris); C.A. Murray (c/o RGO); G. Ruben (Potsdam); H.G. Walter (Hei-

\footnotetext{
43C273B; OQ208; 3C371; 3C309.3.
} 
delberg); G.L. White (c/o CSIRO).

\section{References}

Anderson, B., Argue, A.N., Argyle, R.W., Davis, R., Morrison, L.V., Wilkinson, P.N., 1989.: private communication

Brosche, P., Ducourant, C., Galas, R., Geffert, M., Karafistan, A.: 1990, Astron. Astrophys. (in press)

De Vegt, C., Johnston, K.J., Florkowski, D.: 1990, this Colloquium

Dick, W.R., Ruben, G., Schilbach, E., Scholz, R.-D.: 1987, Astron. Nachr., 308, 21

Feissel, M.: 1990, in Inertial Coordinate System on the Sky, IAU Symp. No 141, Leningrad, October 17-21, 1989, eds.J.H. Lieske and V.K. Abalakin, Kluwer, p. 511

Froeschlé, M., Kovalevsky, J.: Astron. Astrophys. 116, 89

Jefferys, W.H., Benedict, G.F., Duncombe, R.L., Franz, O.G., Frederick, L.W., Hemenway, P.D., Shelus, P.J., Van Altena, W.: 1990, this Colloquium.

Klemola, A.R.: 1990, in Inertial Coordinate System on the Sky, IAU Symp. No 141, Leningrad, October 17-21, 1989, eds. J.H. Lieske and V.K. Abalakin, Kluwer, p. 407

Kumkova, I.I., Tel'njuk-Adamchuk, V.V., Irkaev, B.N., Inasaridse, R. Ya., Bashtova, L.I., Dick, W.H.: 1990, this Colloquium

Lestrade, J.-F., Preston, R.A., Gabuzda, D.C., Phillips, R.B.: 1990, this Colloquium

Morrison, L.V., Argyle, R.W., Requième, Y, Helmer, L., Fabricius, C., Einicke, O.H., Buontempo, M.E., Muinos, J.L., Rapaport, M.: 1990. Astron. Astrophys. (in press)

Russell, J.L., Johnston, K.J., De Vegt, C., Zacharias, N., Ma, C., Shaffer, D., Jauncey, D., Reynolds, J., White, D., Hughes, J., Hindsley, R.: 1990, this Colloquium

Van Altena, W.F., Girard, T., López, C.E., López, J.A., Molina, E.: 1990, in Inertial Coordinate System on the Sky, IAU Symp. No 141, Leningrad, October 17-21, 1989, eds. J.H. Lieske and V.K. Abalakin, Kluwer, p. 419

Walter, H.G.: 1990, Astron. Astrophys. Suppl. Ser. (in press). 